\title{
Research Paper \\ The Role of Impulsivity, Sensitivity to Reward and Anhedonia in Distinction People With Symptoms of Borderline Personality Disorder From Ordinary People
}

\author{
Majid Mahmood Aliloo ${ }^{1}\left(0\right.$, Tooraj Hashemi Nosratabad ${ }^{1},{ }^{*}$ Alireza Karimpour Vazifehkhorani ${ }^{2}$
}

1. PhD in Psychology, Professor, Department of Psychology, Faculty of Education and Psychology, Tabriz University, Tabriz, Iran.

2. MA, Department of Psychology, Faculty of Education and Psychology, Tabriz University, Tabriz, Iran.

\begin{tabular}{|l|l|l}
\hline $\begin{array}{c}\text { Use yourdevice to scan } \\
\text { and read the article online }\end{array}$ & $\begin{array}{l}\text { Cittation: Mahmood Aliloo M, Hashemi Nosratabad T, Karimpour Vazifehkhorani A. [The Role of Impulsivity, Sensitivity to } \\
\text { Reward and Anhedonia in Distinction People With Symptoms of Borderline Personality Disorder From Ordinary People (Per- } \\
\text { sian)]. Iranian Journal of Psychiatry and Clinical Psychology. 2018; 24(2):136-147. http://dx.doi.org/10.32598/ijpcp.24.2.136 }\end{array}$ \\
doi:: http://dx.doi.org/10.32598/ijpcp.24.2.136
\end{tabular}

Received: 15 Oct 2017

Accepted: 26 Feb 2018

Keywords:

Reward sensitivity, Anhedonia, Impulsivity, Reward deficiency syndrome, Sub-threshold borderline personality

\begin{abstract}
Objectives The aim of this study is to determine the role of impulsivity, sensitivity to reward and also anhedonia in distinction People with symptoms of borderline personality disorder from normal people. Methods This study uses both the descriptive and correlative method. The sampling method was sampling and targeting type. The samples comprised of students from Tabriz University (2016-2017 academic year), initial 200 candidates for screening and subsequent 80 candidates for target sampling. In the first step of this study, the Millon Clinical Multiaxial Inventory (MCMI-III) was used for screening followed by Balloon Analogue Risk Task for measuring the impulsivity and resistance variable as sensitivity to reward and the Snaith-Hamilton scale of pleasure is used to measure anhedonia variable.

Results It was illustrated broadly that the impulsivity variable, with 0.61 ratio, has the most important role in the Detection function. The resistance variable towards earning rewards with a 0.47 ratio occupies second place in the Detection function; the anhedonia variable occupied the third place in the Detection function with a ratio of 0.42 .

Conclusion Reward deficiency syndrome can be considered as a fundamental pathological symptom of impulsivity and anhedonia in borderline personality disorder BPD. The poor functioning of the brain's rewards system is a deprivation of sensory mechanisms in people affected with BPD, that led to anhedonia and low arousal and finally impulsivity.
\end{abstract}

\section{Extended Abstract}

\section{Introduction}

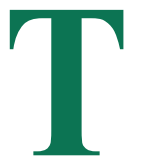

he purpose of this study was to determine the role of impulsivity, rewarding sensitivity and anhedonia between individuals with borderline personality disorder and normal people. According to the theory of reward deficiency syndrome, although impulsive behaviors are quite associated with possible injury or loss, yet they provide the conditions for achieving a type of reward. The reward deficiency syndrome is a psychological theory first noticed by Blum, which is known by the search for rewardseeking behavior or addictions. According to Blum's research, people with reward deficiency syndrome tend to follow the most severe emotions, since it allows them to feel continuous and permanent enjoyment and reward, thereby the dopamine levels increases in their brains. Reward sensitivity, in disorders associated with reward deficiency syndrome, means that individuals do not enjoy the usual rewards seeking process in their natural and daily life activities which leads to various mental disorders, abnormal and resistant patterns of achieving rewards and pleasure.

* Corresponding Author:

Alireza Karimpour Vazifehkhorani, MA

Address: Department of Psychology, Faculty of Education and Psychology, Tabriz University, Tabriz, Iran.

Tel: +98 (912) 9343237

E-mail: a.karimpour92@gmail.com 


\section{Method}

The research method was descriptive-correlational. The statistical population of the present study included the students of Tabriz University in the 2016-17 academic year. According to the research method, 200 people in the first stage were selected by screening, and according to the Tabakhnick \& Fidell formula, 80 were selected by purposeful sampling in the second stage. In fact, according to the mean and standard deviation obtained on the Millon scale-3, 40 people with borderline personality disorder and 40 normal people were selected. In other words, the subjects' scores were converted to the standard $\mathrm{Z}$ score, those who had a score of above Z 1.5 were considered as individuals with borderline personality signs and those with a low Z-negative of 1.5 were normal individuals. In other words, the conversion of raw scores to $\mathrm{Z}$ scores standardized and displayed the scores of subjects in Z's distribution with mean as zero and standard deviation of one in turn controlling the scattering. To measure the variables in the first stage of the study, the Millon-3 questionnaire was used to screen individuals with borderline personality disorder, computerized task of balloon risk for measuring the impulsivity variable and the Essen and Hamilton's pleasure scale for measuring anhedonia variable.

\section{Results}

The results of the study showed that the impulsivity variable with the coefficient of 0.61 has the most effective role in the discriminant function while the sensitivity of reward variable with a coefficient of 0.47 and the anhedonia variable with a coefficient of -0.42 are second and third in order, respectively.

\section{Discussion}

In sum, the results of present study indicate that impulsivity, rewarding sensitivity and anhedonia are the most important factors in differentiating between the individuals with borderline personality disorder and the normal individuals. According to the theory of reward deficiency syndrome, Eysenck's, Gary's, and Zuckerman's, it can be said that these three characteristics have a high diagnostic value in individuals with borderline personality traits. As per Eysenck's theory, people with higher emotional instability (such as borderline personality) are more sensitive to threats and reward signs/clues, thus because of anhedonia, they look for excitement and incitation in the environment. According to Gary's theory, people with high impulsivity have a higher sensitivity in the BAS system which makes them more capable/susceptible to potential rewards symptoms and have a low inhibitory mechanism, seen in addictive and enthusiastic behaviors. On the other hand, according to Zuckerman's theory, seekers of superior senses are in a state of anhedonia, therefore need more powerful stimuli to achieve optimal levels of arousal. He also stated that while facing with challenging and reward-based task, responses are more severe.

Thus, according to the theory of reward deficiency syndrome, a defect in the brain reward system is a kind of deprivation of sensory mechanisms in individuals with signs of borderline personality. This leads to anhedonia, low arousal and finally leads to impulsivity in such individuals. However, more studies are needed to clarify the role of the reward deficiency syndrome in borderline personality disorder. In general, the results of the present study can be inferred that anhedonia can be considered as an underlying factor in the incidence of impulsive behaviors and abnormal patterns of reward seeking in people with borderline personality signs. However, the clarification of this issue requires further researches.

\section{Ethical Considerations}

\section{Compliance with ethical guideline}

The participants signed the informed consent form and had the right to leave the study at any time.

\section{Funding}

This research did not receive any specific grant from funding agencies in the public, commercial, or not-for-profit sectors. The paper was extracted from the last author MA thesis in the Department of Psychology, Faculty of Education and Psychology, Tabriz University.

\section{Conflict of interest}

There authors declare that they have no conflict of interest.

\section{Acknowledgements}

In the end, we thank all the participants who helped us complete this study. 


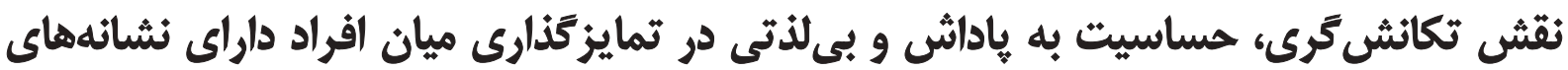

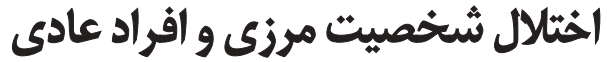

\author{
مجيد محمودعلى لو'، تورج هاشمى نصرتآباد'، “عليرضا كريميور وظيفهخورانى' \\ 1 - د دكتراى روانشناسى، استاد، كروه روانشناسى، دانشكده علوم تربيتى و روانشناسى، دانشعاه تبريز، تبريز، ايران.

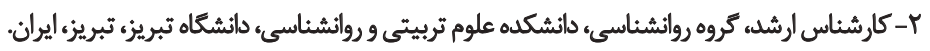

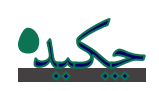

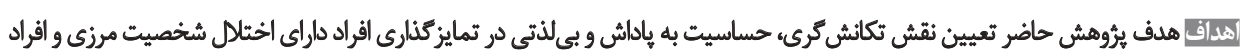
:

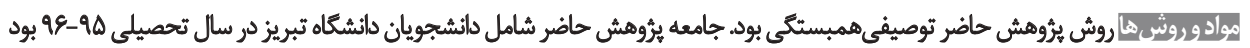

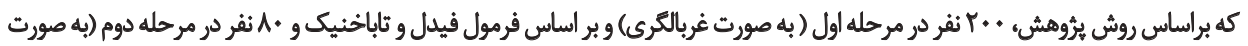

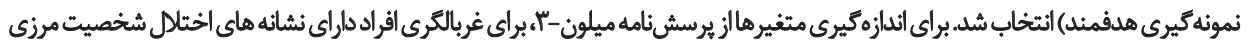

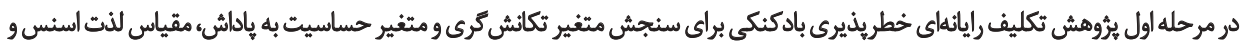

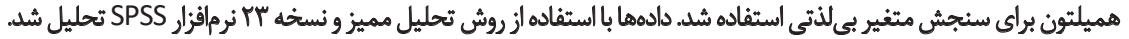

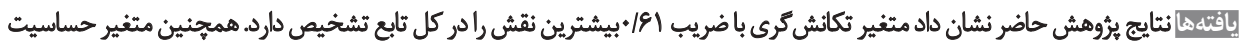

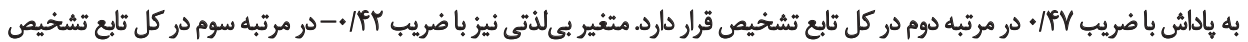
قرار دارد.

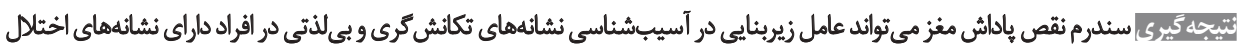

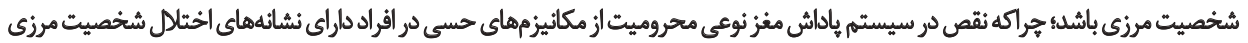

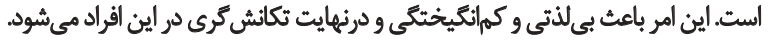

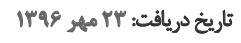

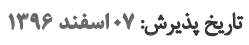
كليدوازوها:

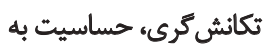

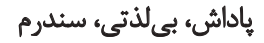 نقص باداش مغز، اختيلال شخصيث هرزي باد مغن

در كنترل خشم و علائم هارانوئيدى و تجزيهاى كثذرا از نشانهاي

مقام

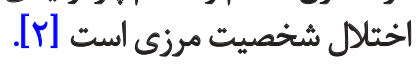

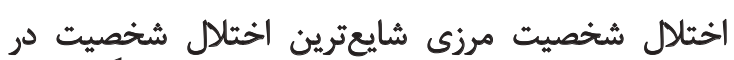

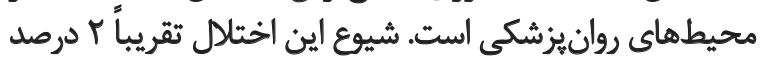

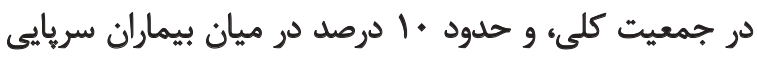

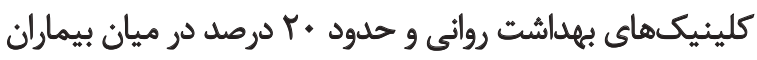

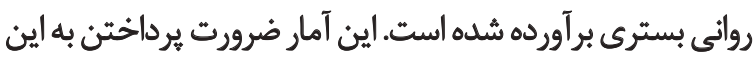

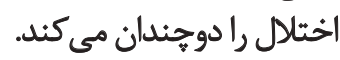

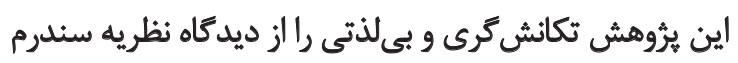

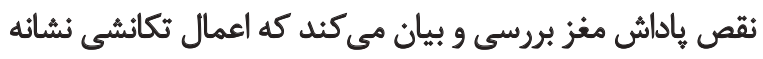

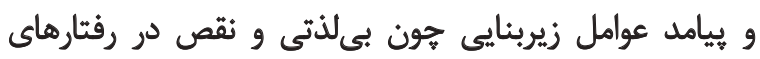

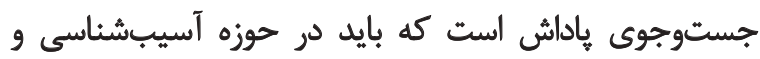

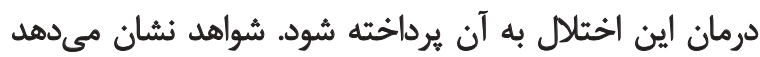
عوامل عصبشناختى با اختلال شخصيت مرزى ارتباط دان دارند

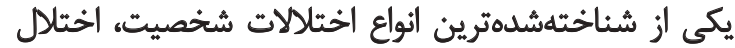

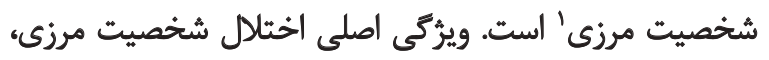

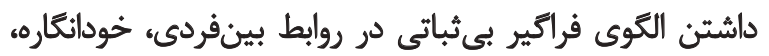

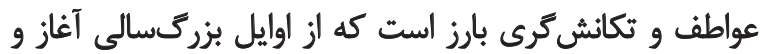

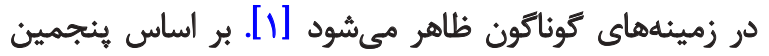

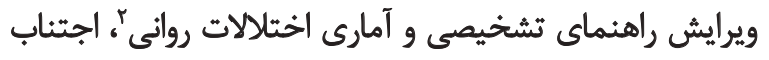

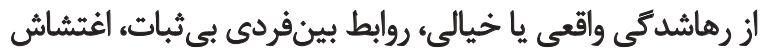

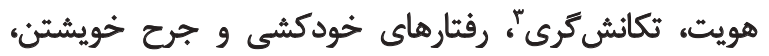

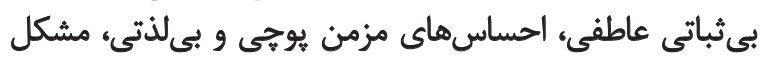
1. Borderline Personality Disorder (BPD)
2. Diagnostic and Statistical Manual of Mental Disorders-5 (DSM-5)
3. Impulsivity

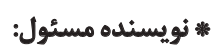

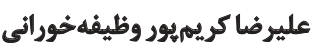

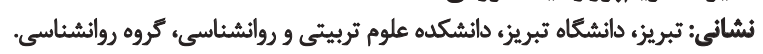
تلفن: a.karimpour92@gmail.com يست الكترونيكي: 
(برائكيختىى بيش از حل معمول) در بيماران مبتلا به اختلال

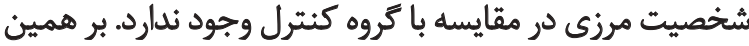

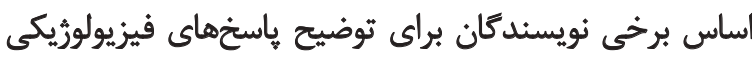

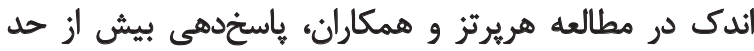

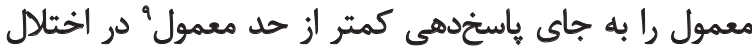

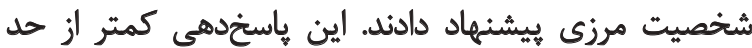

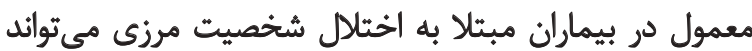

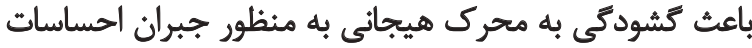

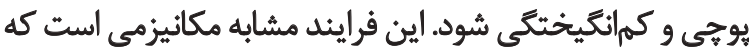

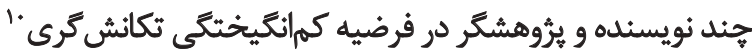

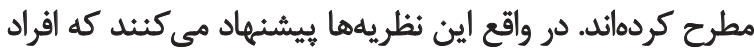

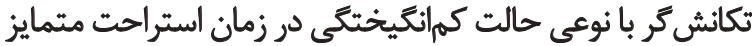

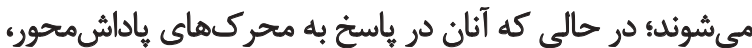

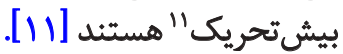

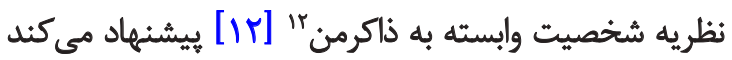

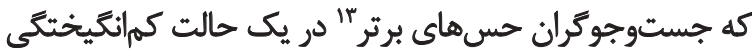

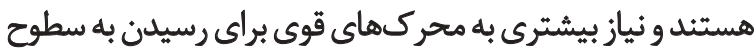

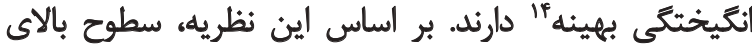

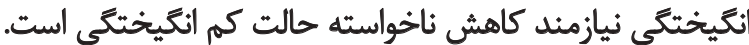

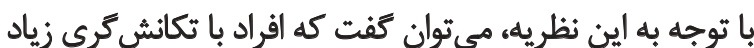

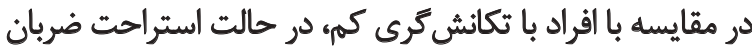

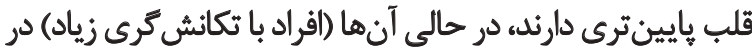

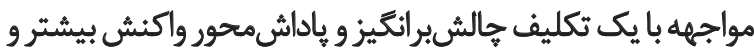

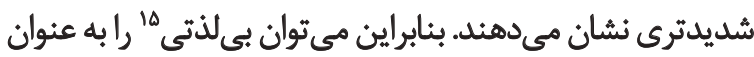

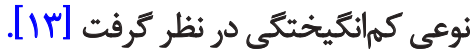

هدف اصلى يروهش حاضر تعيين نقش تكانش گرى، حساسيت

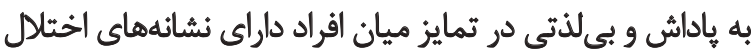

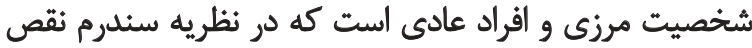

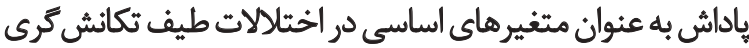

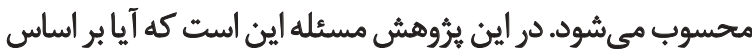

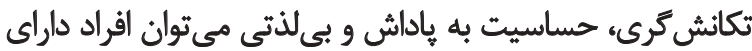

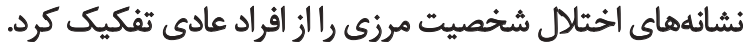

فرضيههاى يُروهش حاضر عبارتند از: تكانش ترى مى ثواند افراد

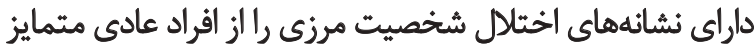
كند؛ حساسيت به بِاداش مي ثواند افراد داراي نشانههاي اختلايل

\footnotetext{
8. Hyperarousal

9. Hyperresponsiveness \& hyporesponsiveness

10. Underarousal hypotheses of impulsivity

11. Over aroused

12. Zuckerman

13. High sensation seekers

14. Optimal level of arousal

15. Anhedonia
}

همجينين متغيرهاى بيولورُيكى باشخصيت مرزى هرتبط هستند. اين عوامل در ضرورت انجام اين يروهش نقش مهين مهمى دارند. يكى از نشانههاي اصلى در افراد داراى نشانههاى اختلال

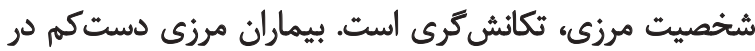

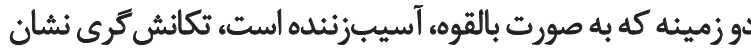

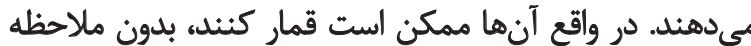

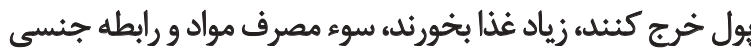

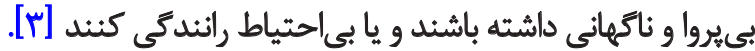

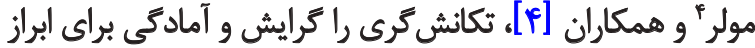

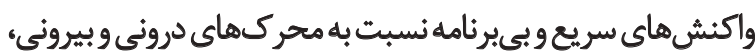

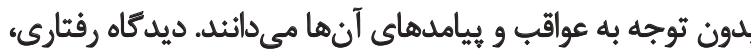

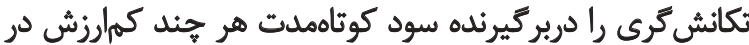

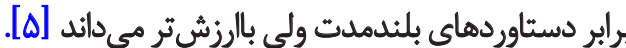
براساس نظريه سندرم نقص ياداش مغزه هفتارهاى تكانشى

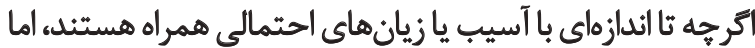

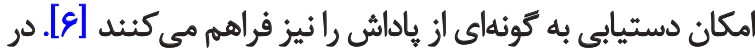

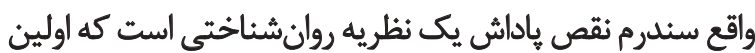

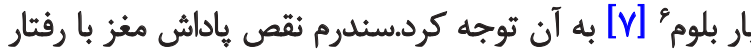

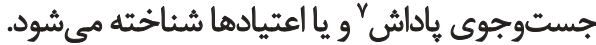
براساس تحقيقات بلوم [ᄉ] افراد مبتلا به سندرم نقص يّاداش

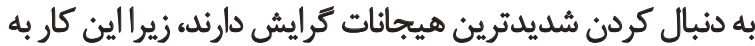

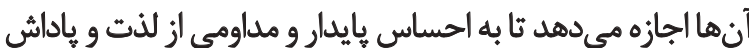

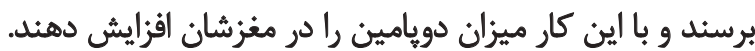

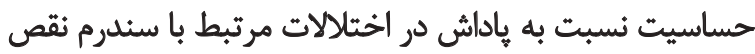

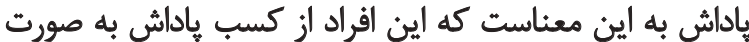

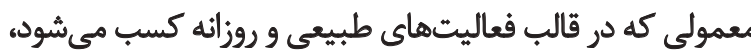

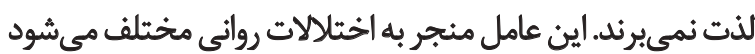

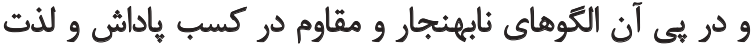

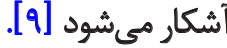

با توجه به اينكه يكى ديكر از نشانههاي افراد داراي اختلال

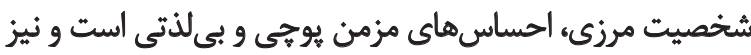

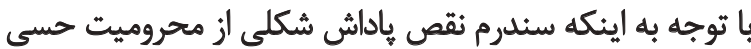

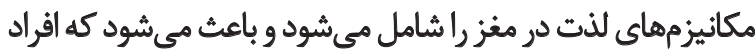

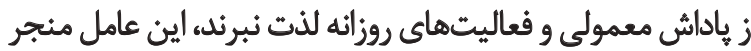
به اختلالات روانى مانئد اختلال شخصيت مرزى مى مشود [ـ [1]. بررسى ميزان ضربان قلب، فشار خون و ياسخ يوست

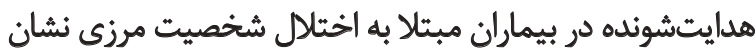
داد هيج شاهدى از بيشانكيختكى در حالت عادى و استراحت

4. Moeller

5. Reward deficiency syndrome

6. Kenneth Blum

7. Reward-seeking behavior 


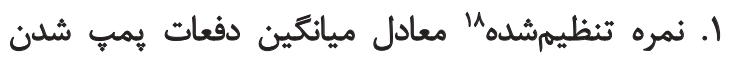

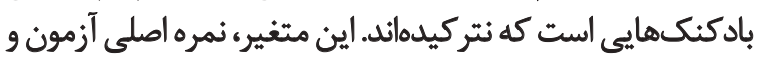

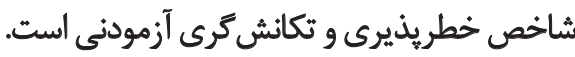

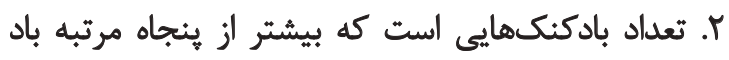

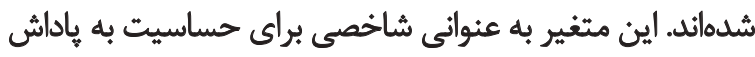

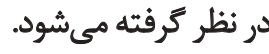

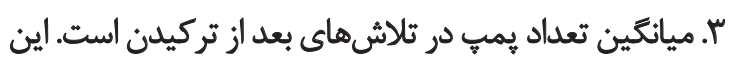

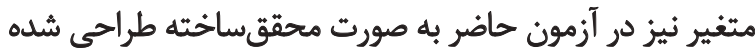

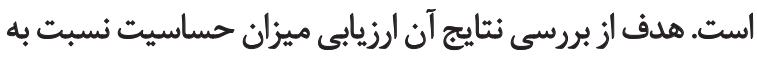

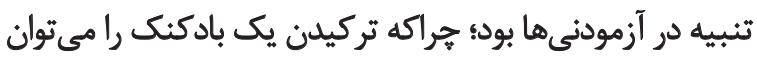

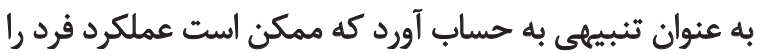
براى بادكنك بعدى تحت تأثير قرار دهد.

$$
\text { مقياس سنجش بى لذتى }
$$

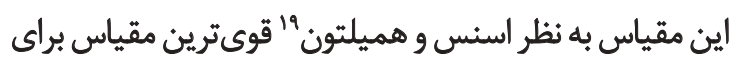

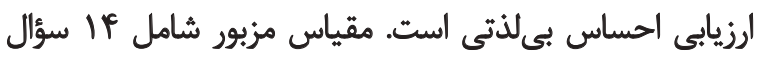

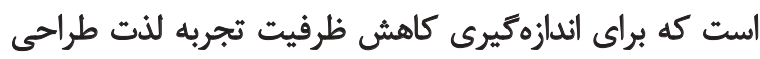

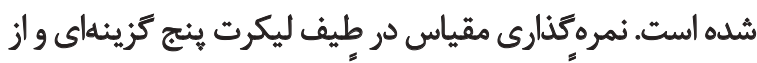

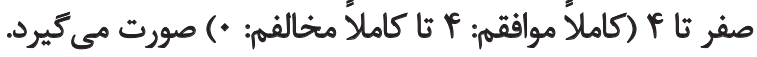

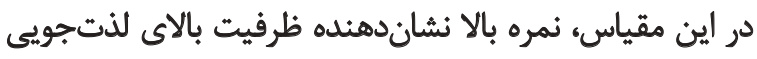

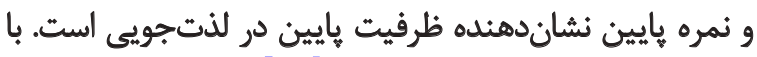

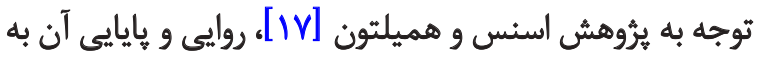

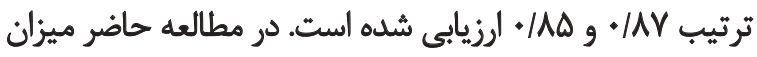

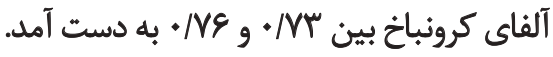

\section{برسش نامه ميلون-r}

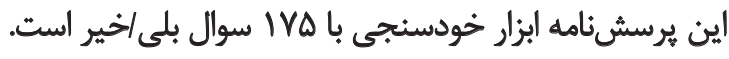

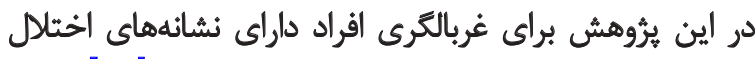

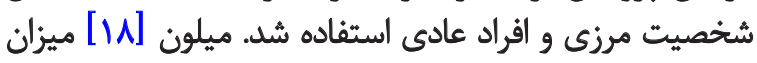

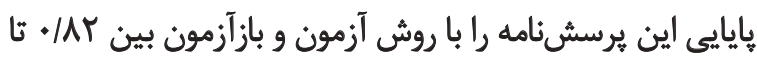

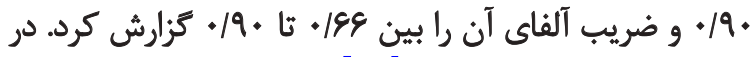

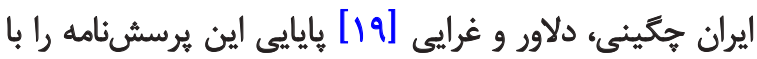

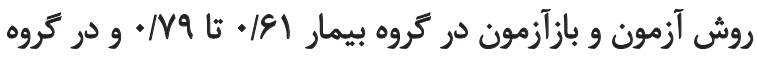

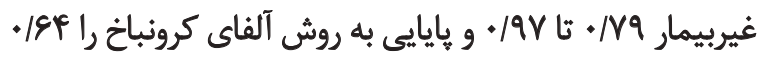

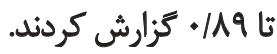

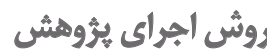

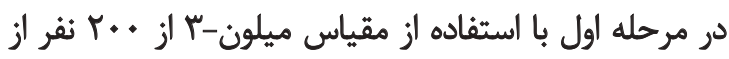

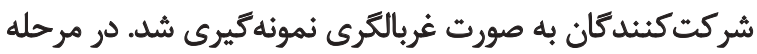

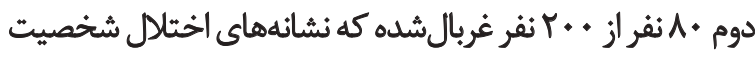

18. Adjusted value

19. Snaith - Hamilton Pleasure

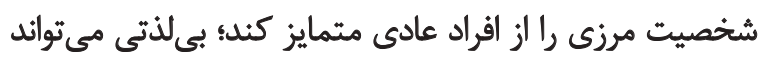

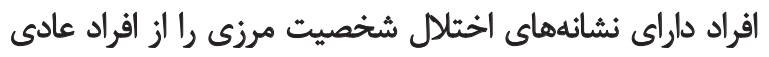

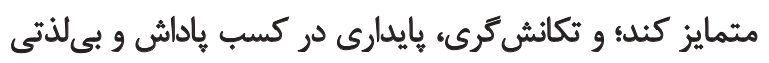

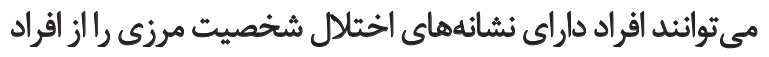

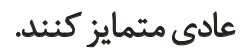

وش - موش

روش يُروهش حاضر توصيفى همبستكى بود. جامعه يُروهش،

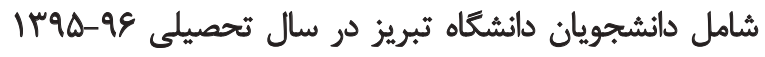

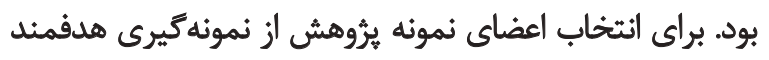

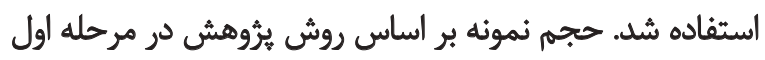

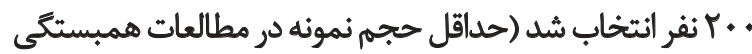

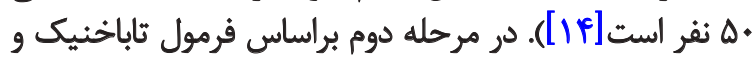

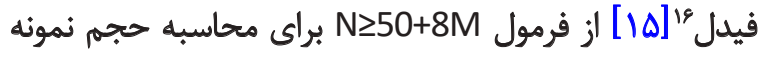

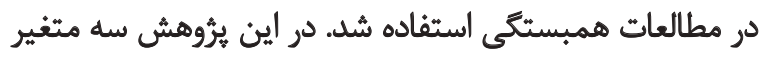

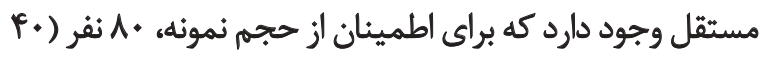

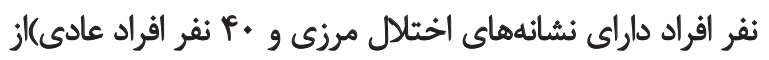

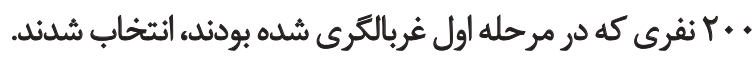
ملاك هاى ورود به مطالعه عبارت بودند از: داشتن دامنه سنى

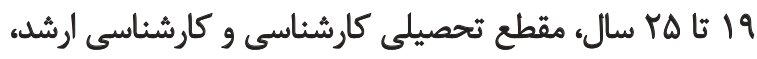

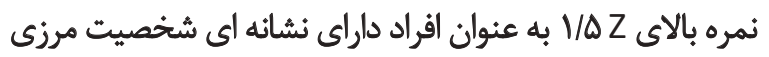

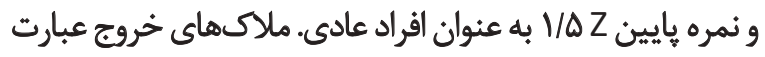

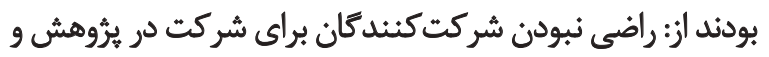
مبتلانبودن به اختلالات روائي.

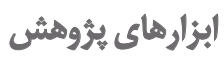
أزمون رايانهاى خطريذيرى بادكنكى براى سنجش تكانش بـرى و حساسيث به ياداش

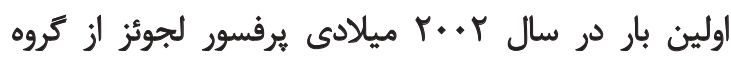

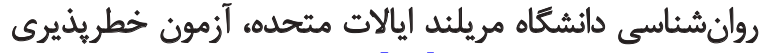

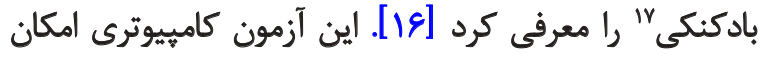

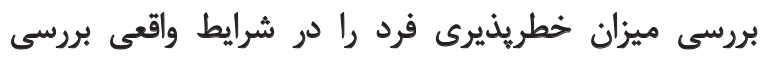

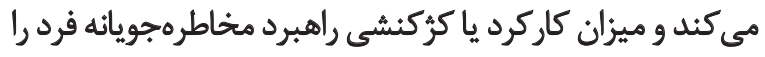

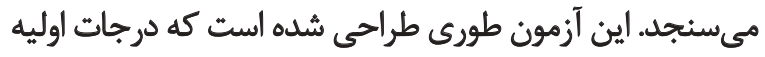

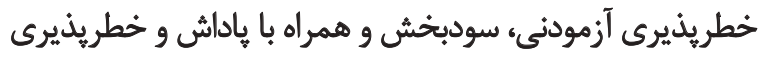

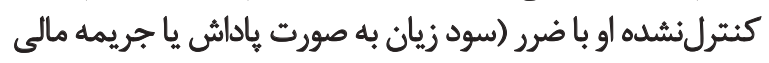

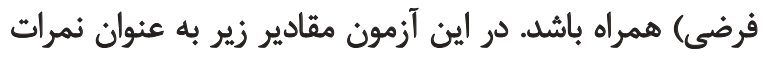

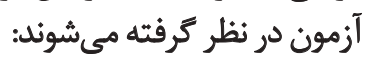

17. Balloon Analogue Risk Task (BART) 


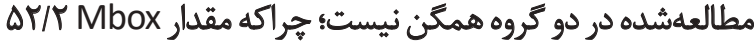

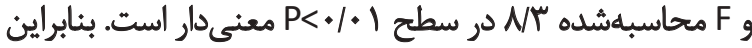
بيشفرض همكنى ماتريسهاى واريانس و كوراريانس محقى نشده است.

جدول شماره Y نشان مىدهد كه اجراى تحليل مميز

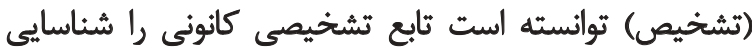

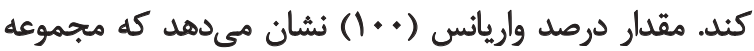

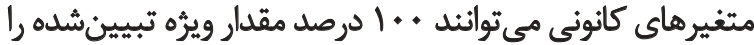

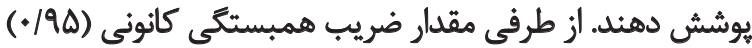

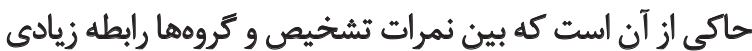

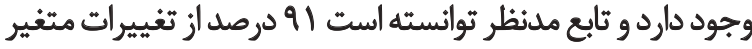

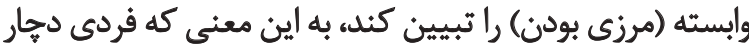
اختلال شخصيت مرزى خواهد شد يانه.

نتايج آزمون آماره لانداى ويلكس نشان مى دهد حدود 9 درصد

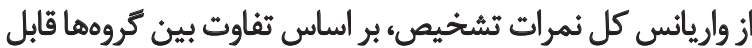

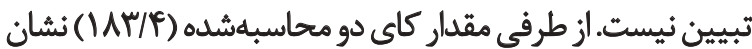

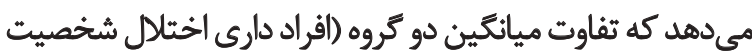

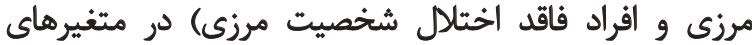

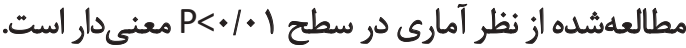
آزمون ضرايب استانداردشده تابع تشخيص كانونى نشان داد كه تكانش

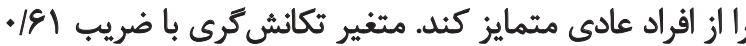

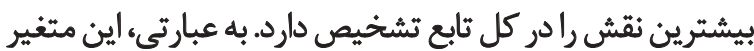

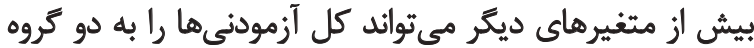

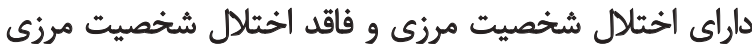

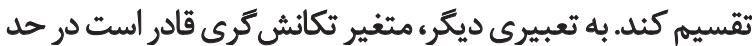

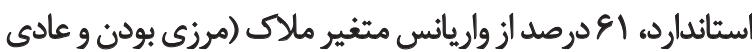
بودن) را به صورت مثبت تبيين كند.

همجنين آزمون ضرايب استانداردشده تابع تشخيص كانونى

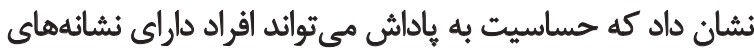

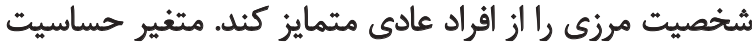

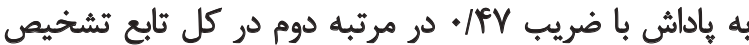

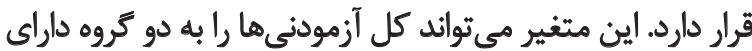

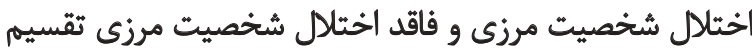

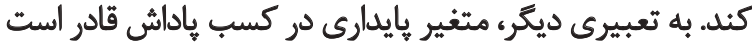

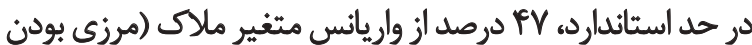
و عادى بودن) را به صورت مثبت تبيين كند.

آزمون ضرايب استانداردشده تابع تشخيص كانونى نشان داد

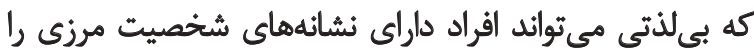

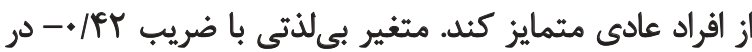

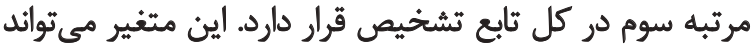

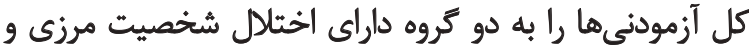

مرزى و عادى را داشتثلد براى شركت در مرحله اصلى يُرؤهش

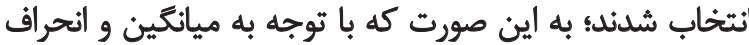

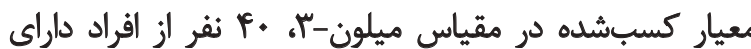

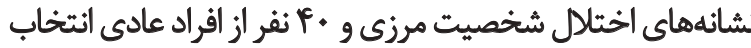

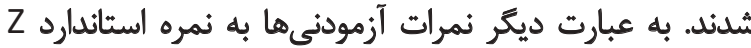

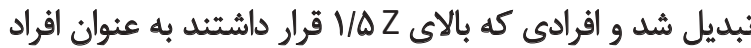

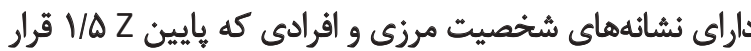

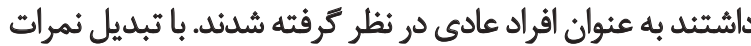

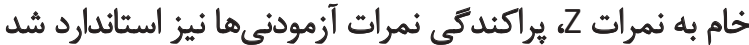

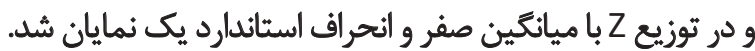

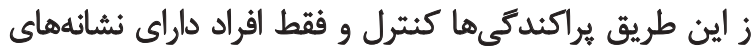
اختلال شخصيت مرزى براساس نمرات Z غربال شدند.

براى رعايت ملاحظات اخلاى يُوهش يُ إز بئ بيان اهداف

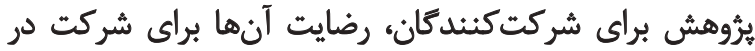

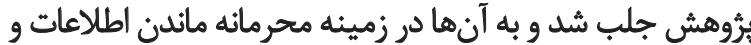

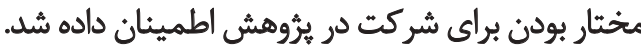

تحليل دادهها

براى تجزيهوتحليل دادههادر بخش آمار توصيفى از شاخصهاى

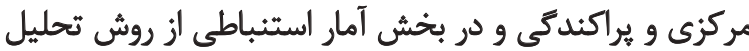

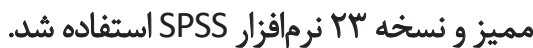

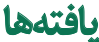

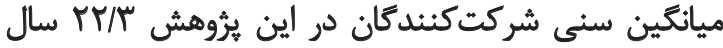

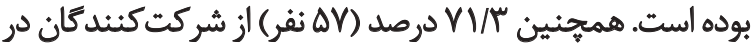

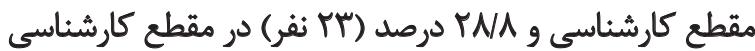

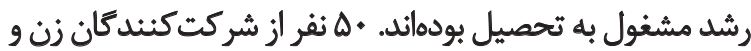

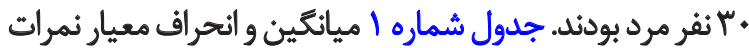

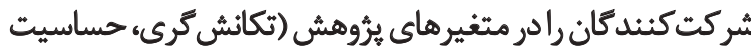

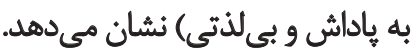

نتايج آزمون برابرى ماتريس كوواريانس متغيرها (لكاريتم

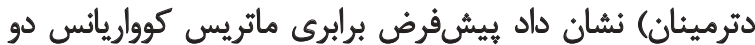

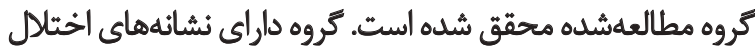

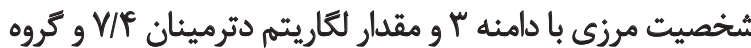

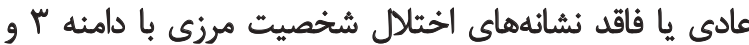

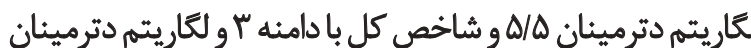

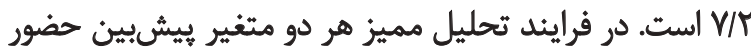

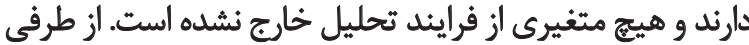

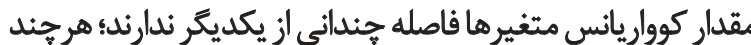
كه مقدار كوواريانس در كروه اول كمى بيشتر از كروه دوم است.

نتايج آزمون همكنى ماتريس واريانس و كوواريانس متغيرها

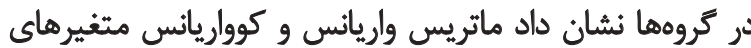


جدول ا. ميانتين و انحرافمعيار متغيرهاي يُروهش

\begin{tabular}{|c|c|c|c|}
\hline تعداد & ميانكين (انحراف معيار) & \multicolumn{2}{|c|}{ مثغير } \\
\hline f. & $r V / F(r / S)$ & تكانش كرى & \\
\hline f* & 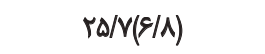 & حساسيت به ياداش & شخصيت مرزى \\
\hline f. & $r / \Psi(r / *+\Lambda)$ & بى للأتى & \\
\hline r. & $\Delta / \Delta(Y / \Delta)$ & تكانش كرى & \\
\hline r. & $g / Q(T / M)$ & حساسيت به باداش & عادى \\
\hline r. & II/T(TM) & بي للأتى & \\
\hline 1. & $\mid W / F(N \Delta)$ & تكائشكرى & \\
\hline A. & $\mid \varepsilon / \pi(1 \cdot M)$ & حساسيت به باداش & كل - مل \\
\hline ᄉ. & $V / T(F / g)$ & بي لألتي & \\
\hline
\end{tabular}

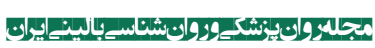

شخصيث مرزى با كنترل اثرات مثغيرهاى ديخر نشان مي دهد. مبتنى بر اين ضرايب، ثنابع تشخيصى به شرح زير خير خواهد بودي

$D F=a+b|X|+b r X r+b r x r$

$D F=-r / \Lambda+(\cdot / / 9) X I+(\cdot / \cdot 9) X r+(\cdot / / V) X r$

بر اساس تابع تشخيصي بهدست آمده مي توان استنباط كرد

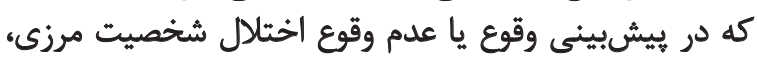

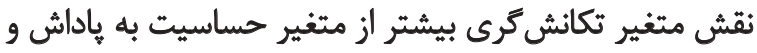
متغير بى للذتى است.

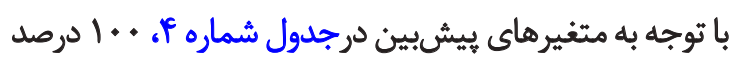

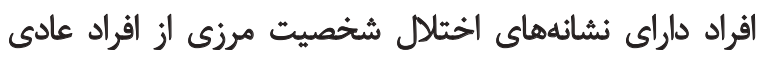

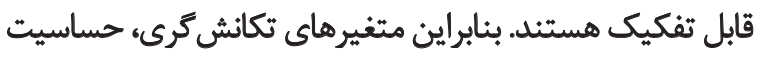

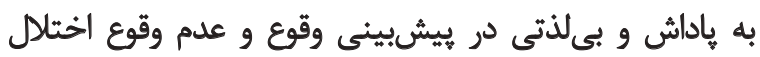

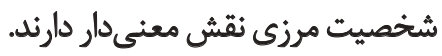

ثک

يُروهش حاضر با هدف تعيين نقش تكانش

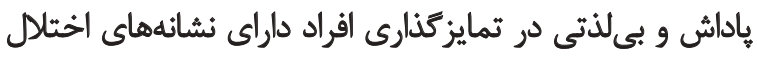

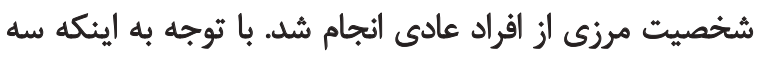

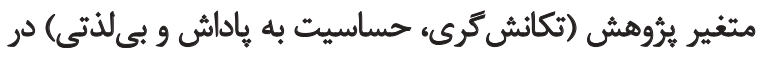

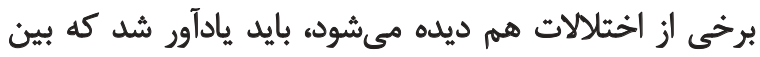

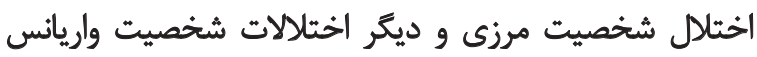

فاقد اختلال شخصيت مرزى تقسيم كند. به تعبير ديكره مثغير

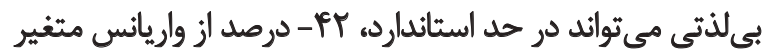
ملاك (مرزى بودن و عادى بودن) را به صورت منفى ثبيين كند. آزمون ضرايب استانداردشده تابع تشخيص كانونى نشان داد كه تكائش

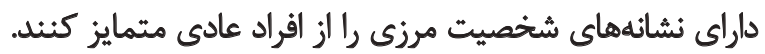

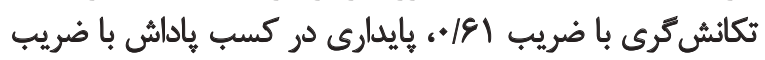

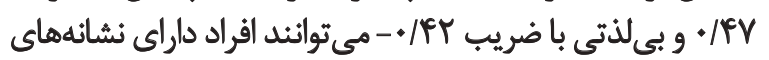
شخصيت مرزى را از افراد بهنجار متمايز كنئد.

آزمون ماتريس ضرايب ساختارى مثغيرها نشان داد كه

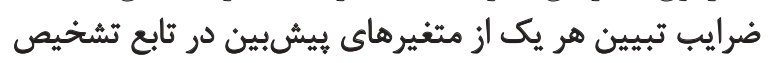

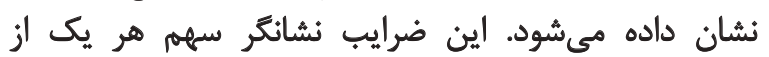

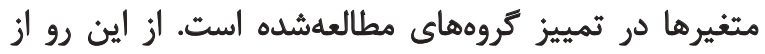
ضرايب ساختارى موجود در جدول مذكور مى توان استنباط كرد كه متغير تكانش

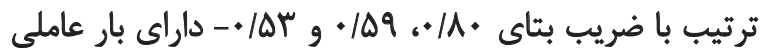

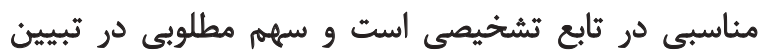

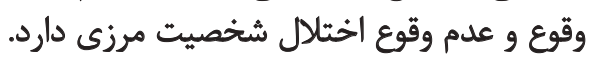
جدول شماره ب ضرايب غير استاندارد ثابع تشخيص كانونى

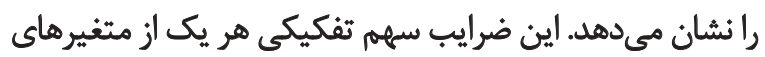

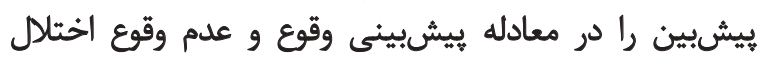

جدول r. مقادير ويرُه ثابع تشخيص كانوئى

\begin{tabular}{|c|c|c|c|c|}
\hline همبستيّى كانونى & ورصد تجمعى & ور صد واريانس & مقدار ويؤه & تابع \\
\hline.$/ 90$ & $1 .+$ & $1 \ldots$ & $1.1 .+1$ & 1 \\
\hline
\end{tabular}

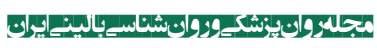


جدول س. ضرايب غيراستاندارد تابع تشخيصى كانوئى

\begin{tabular}{|c|c|c|c|c|}
\hline مقدار ثابت & بى لذتى (XY) & حساسيت به ياداش (XY) & تكانش ترى (X1) & متغير \\
\hline$-T / A$ & $-+/ I V$ & .1 .9 & $+/ 19$ & ضرايب \\
\hline
\end{tabular}

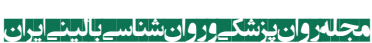

ويشبينى كثنده سايكوياتولوزى مرزى در مرحله بييخيرى بود.

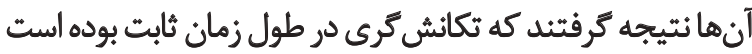

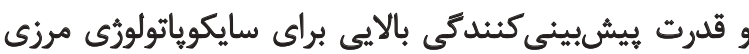

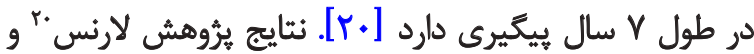

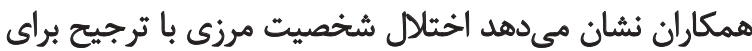

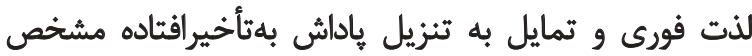

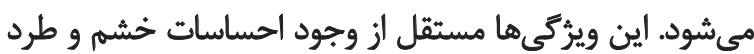

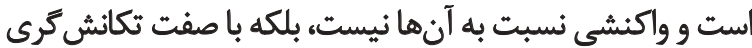
مرتبط است [MI]

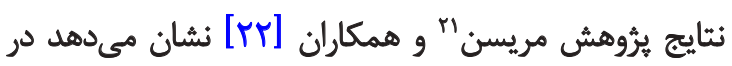

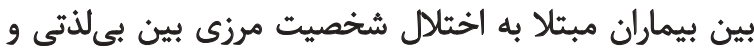

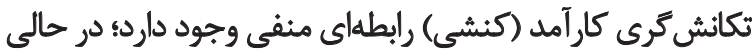

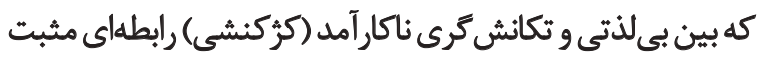

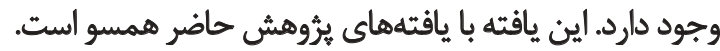
در تبيين يُروهش حاضر مي توان كفت كه بر اساس نظريه

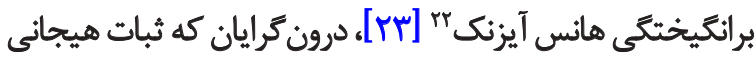

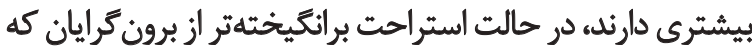

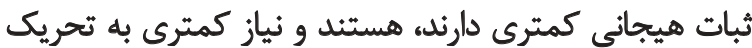

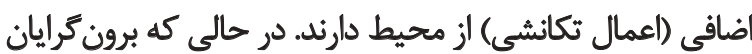

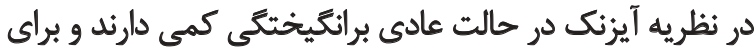

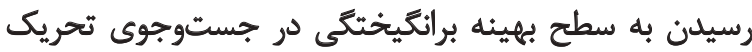

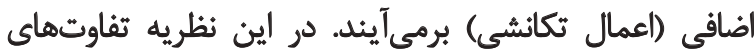

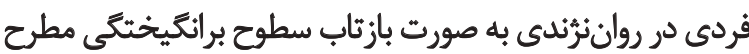

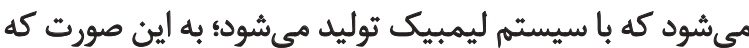

20. Lawrence

21. Marissen

22. Eysenck

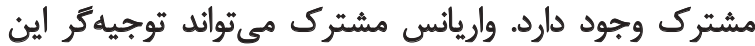

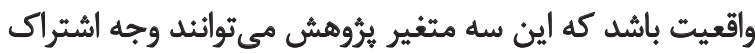
اختلال شخصيت مرزى و ديكر اختلالات باشند.

در تحليلها هم اشاره شده است كه ويزّكىهاى مذكور

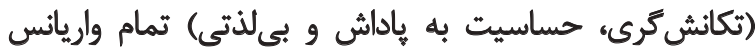

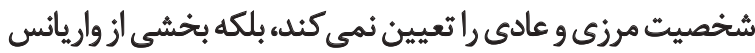

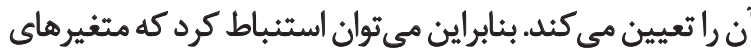

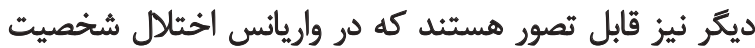

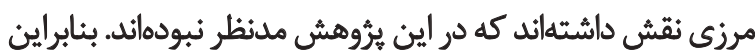

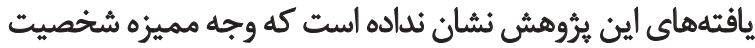

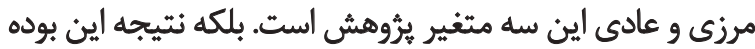

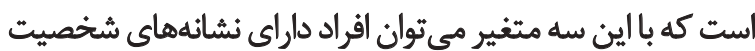
مرزى و عادى را از هم تفكيك كرد. در اين راستا، نخستين يافته اين يُروهش نشان داد داد كه

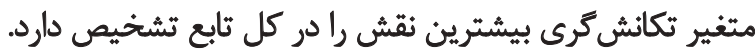

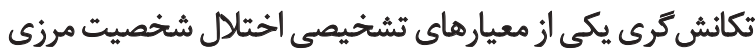

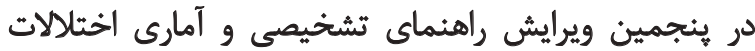

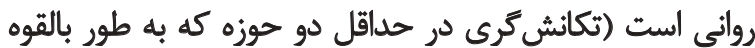

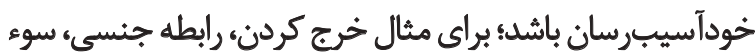

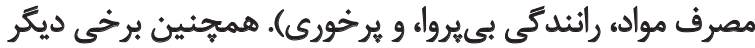

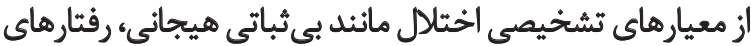

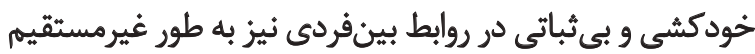

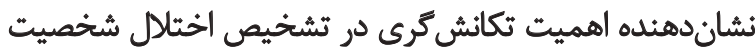

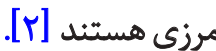

در يروهشى به بررسى اين موضوع برداخته شد كه كدام جنبه

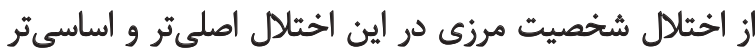

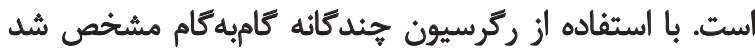
كه نمره زيرمقياس (عمل تكانه) مصاحبه تشخيصى بهترين

جدول F. جدول فراوائى تفكيك كروهها بر اساس متغيرهاى بيشبين

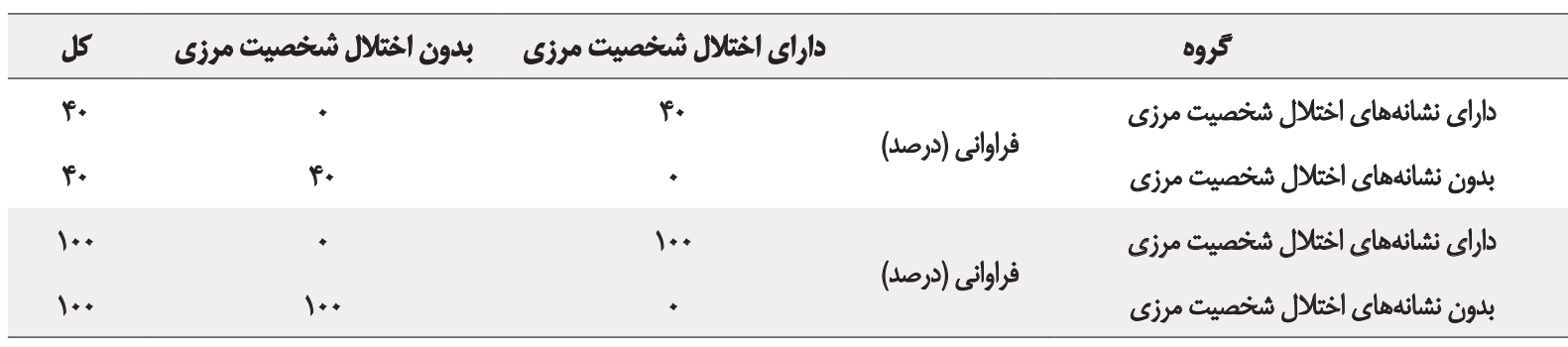




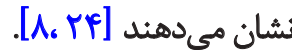

يافتههاى يُروهش حاضر نشان داد متغير بى لذانى در مرتبه

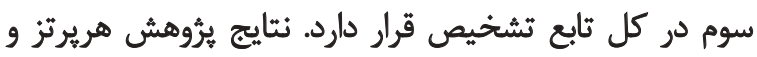

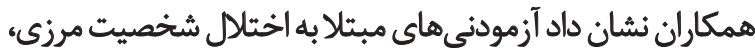

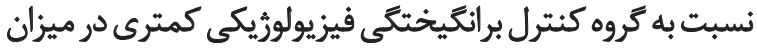

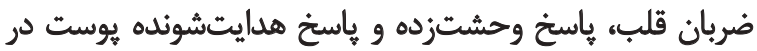

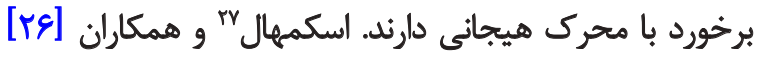

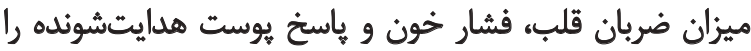

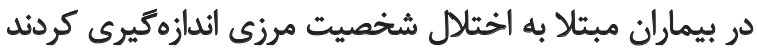

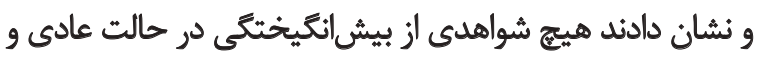

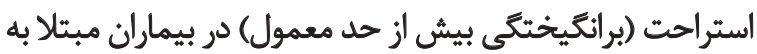
اختلال شخصيت مرزى در مقايسه با كروه كنترل وجود برد بندارد.

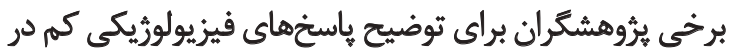

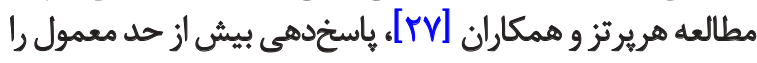

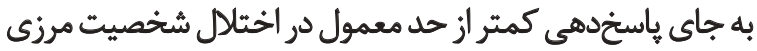

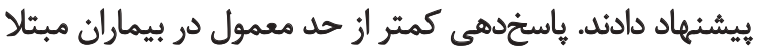

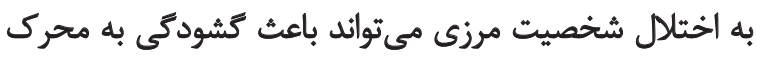

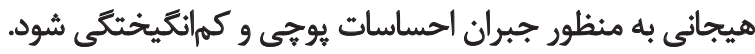

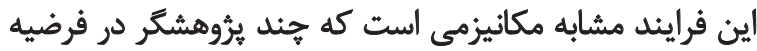

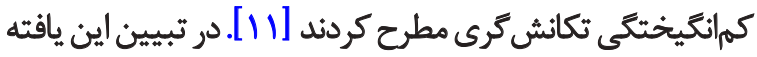

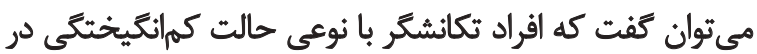

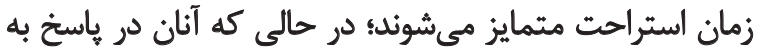

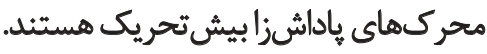

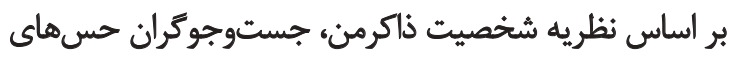

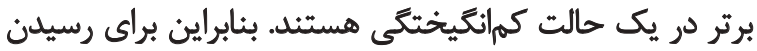

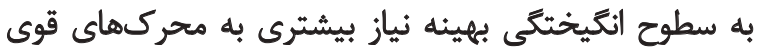

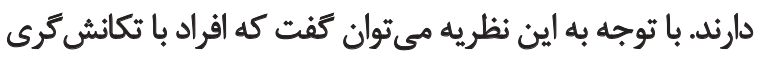

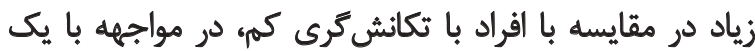

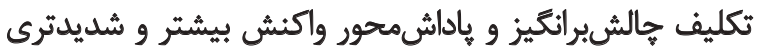

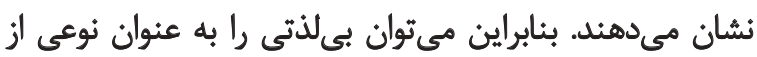
كمانكيختىى در نظر كرفت

براساس نظريه آيزئك [rr]، افراد با نوروتيسيزم زياد كه

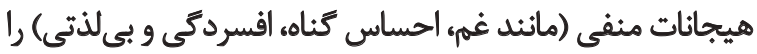

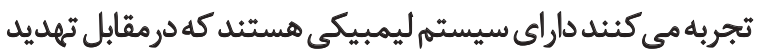

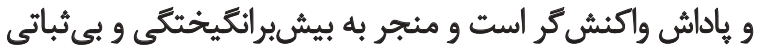

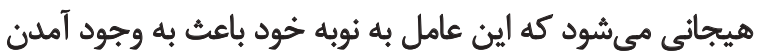

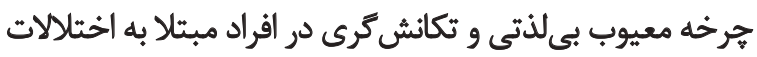

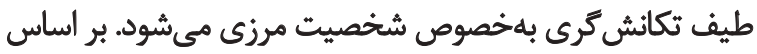

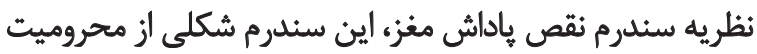

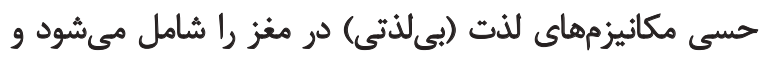

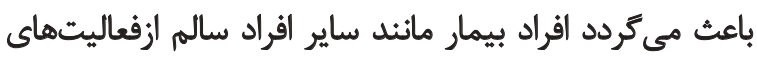

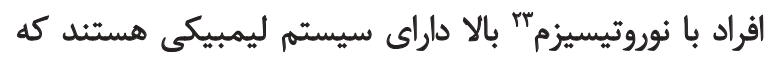

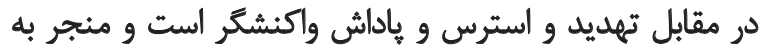

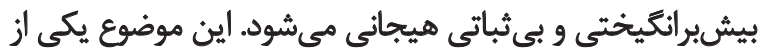

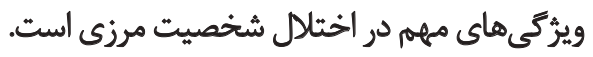

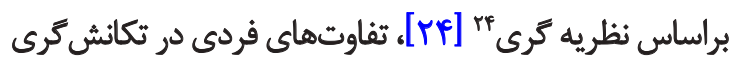

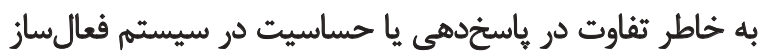

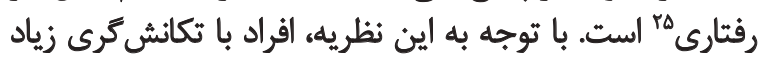

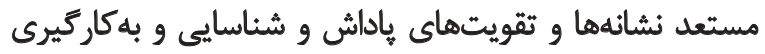

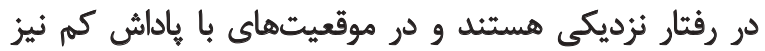

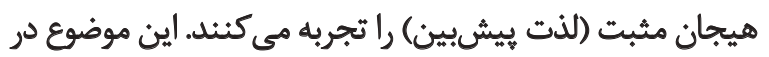

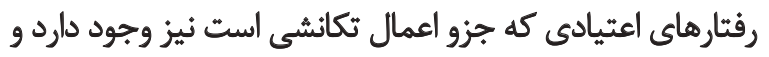

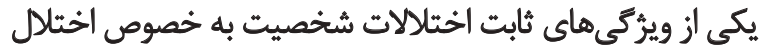

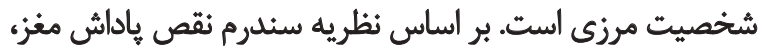

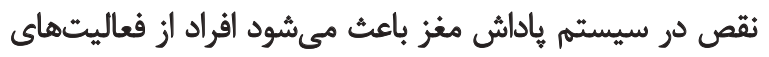

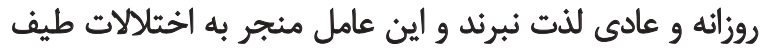

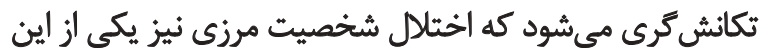

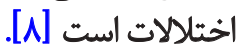

يثروهش حاضر نشان داد متغير حساسيت به باداش در مرتبه باديه

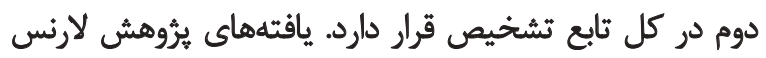

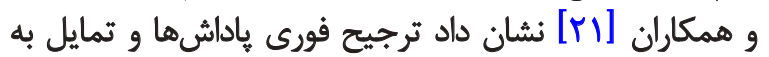

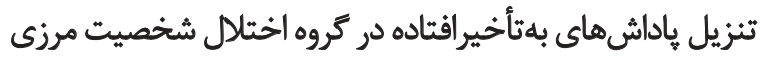

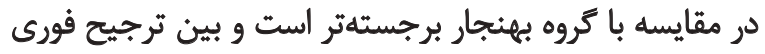

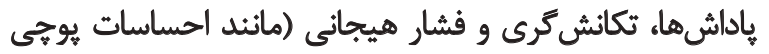

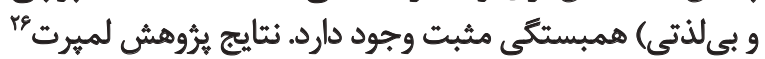

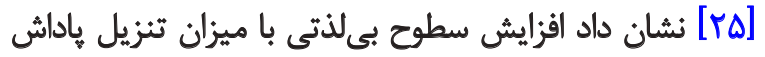

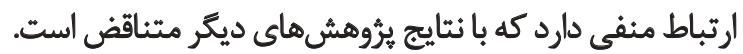

براساس نظريه سندرم نقص باداش مغز در تبيين اين فرضيه

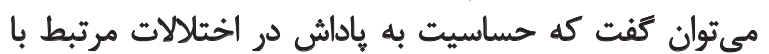

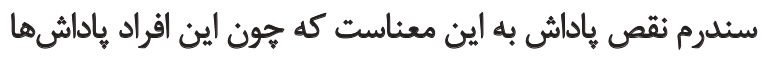

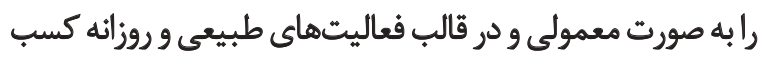

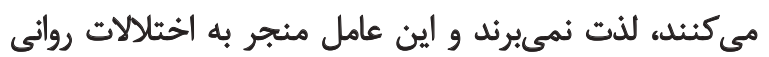

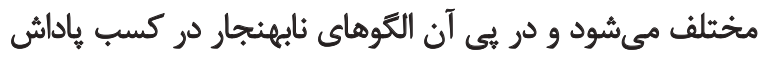

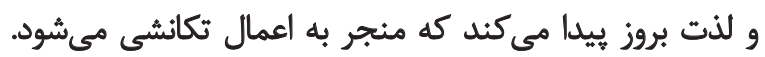

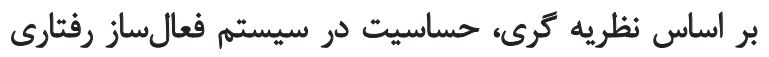

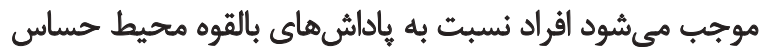

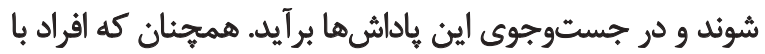

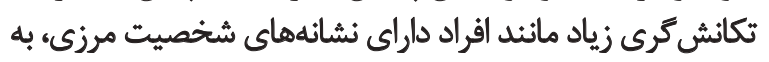

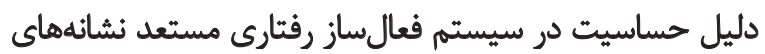

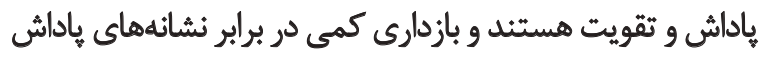

23. Neuroticism

24. Gray

25. Behavioral Activation System (BAS)

26. Lempert 
سباسكّأإى

در عايان از تمامى شركت كنندكان محترم كه ما را در تكميل

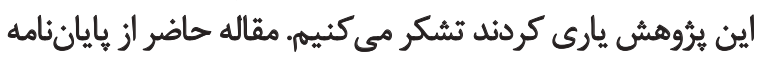

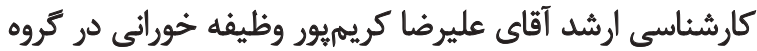

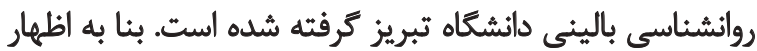
نويسنده مسئول مقاله، تعارض منافع وجود ندئن نداشته است.

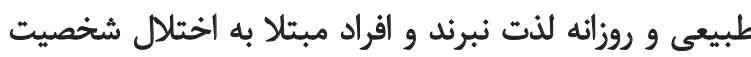

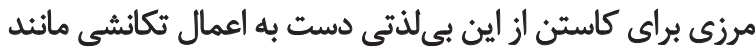

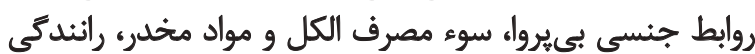
مخاطرهآميز و ساير اعمال تكانشى مى برنئد.

\section{تئيجدكَيرى}

نتايج يروهش حاضرنشان دادمتغيرهاى تكانش ترى، حساسيت

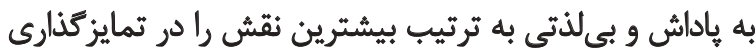

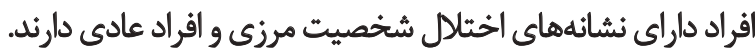

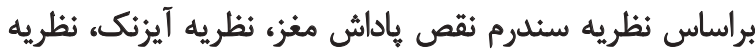

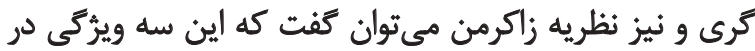

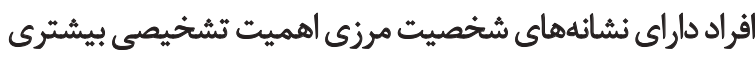

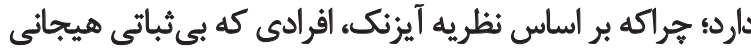

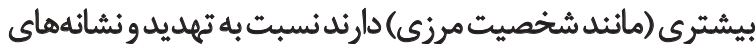

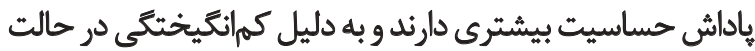

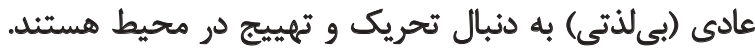

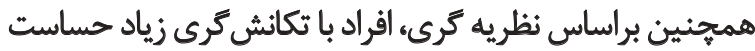

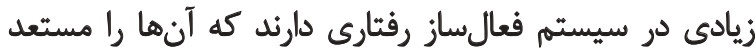

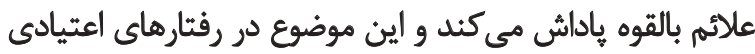

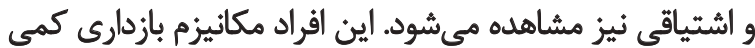

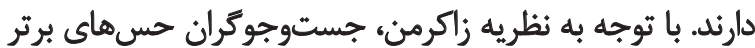

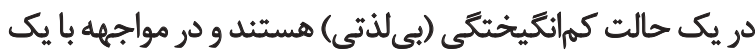

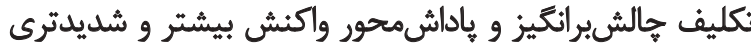
نشيان مي هندئ.

با توجه به نظريه سندرم نقص ياداش مغز، نقص در سيستم

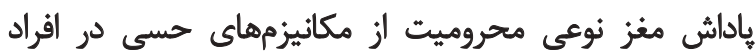

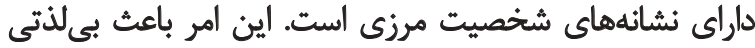

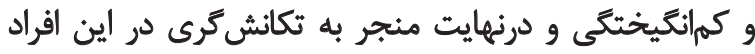

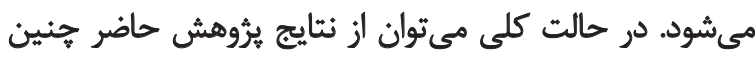

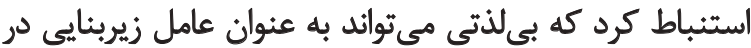

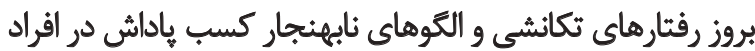
داراى نشانههاى شخصيت مرزى محسوب شودي.

اين يُروهش روى افراد داراى نشانهاي اختلال شخصيت

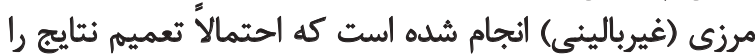

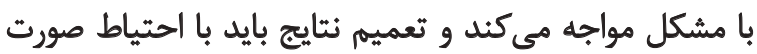

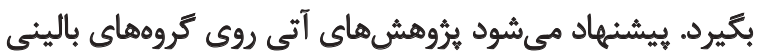

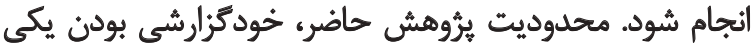

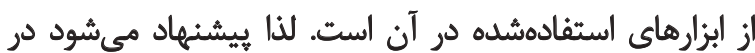

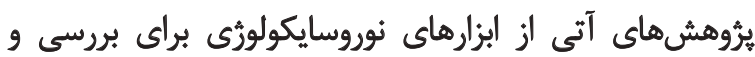
جمع آورى بهتر اطلاعات استفاده شود. 


\section{References}

[1] Mahmoud AM, Sharifi MA. [Borderline personality disorder theoretical topics and therapies (Persian)]. Tehran: Arjmand Publications; 2015

[2] Kaplan HI, Sadock BJ. Kaplan and Sadock's synopsis of psychiatry: Behavioral sciences/clinical psychiatry. Philadephia: Williams \& Wilkins Co; 1998.

[3] American Psychiatric Association. Diagnostic and statistical manual of mental disorders. Washington D.C.: American Psychiatric Press; 2000.

[4] Moeller FG, Barratt ES, Dougherty DM, Schmitz JM, Swann AC. Psychiatric aspects of impulsivity. American Journal of Psychiatry. 2001; 158(11):1783-93.[DOI:10.1176/appi.ajp.158.11.1783]

[5] Petry NM. Substance abuse, pathological gambling, and impulsiveness. Drug and Alcohol Dependence. 2001; 63(1):29-38. [DOI:10.1016/S0376-8716(00)00188-5]

[6] Ekhtiari H, Rezvanfard M, Mokri A. Impulsivity and its Different Assessment Tools: A Review of View Points and Conducted Researches. Iranian Journal of Psychiatry and Clinical Psychology. 2008; 14(3):247-257

[7] Justin Modestino E, Blum K, Oscar-Berman M, Gold MS, Duane DD, Sultan SG., et al. Reward deficiency syndrome: Attentional/ arousal subtypes, limitations of current diagnostic nosology, and future research. Journal of Reward Deficiency Syndrome. 2015; 1(1):6-9. [DOI:10.17756/jrds.2015-002]

[8] Blum K. Substance use disorder a bio-directional subset of reward deficiency syndrome. Frontiers in Bioscience. 2017; 22(9):1534-48. [DOI:10.2741/4557]

[9] Blum KE, Kozlowski GP. Ethanol and neuromodulator interactions: A cascade model of reward. Alcohol and Behavior. 1990; 131-49.

[10] Blum K, Meshkin B, Downs BW. DNA based customized nutraceutical "gene therapy" utilizing a genoscore: A hypothesized paradigm shift of a novel approach to the diagnosis, stratification, prognosis and treatment of inflammatory processes in the human. Medical Hypotheses. 2006; 66(5):1008-18. [DOI:10.1016/j. mehy.2005.09.029]

[11] Savage J. The development of persistent criminality. Oxford: Oxford University Press; 2009. [DOI:10.1093/acprof:o so/9780195310313.001.0001]

[12] Zuckerman M. The psychobiological basis of personality. In Nyborg H, editor. The scientific study of human nature. New York: Elsevier Science; 1997.

[13] Loas G, Pierson A. Anhedonia in psychiatry: A review. MedicoPsychological Annals. 1989; 147(7):705-17. PMID: 2694884

[14] Delavar A. [Research methods in psychology (Persian)]. Tehran: Virayesh Publications; 2006.

[15] Tabachnick BG, Fidell LS. Using multivariate statistics. Boston: Allyn \& Bacon/Pearson Education; 2007. [PMCID]

[16] Rao H, Korczykowski M, Pluta J, Hoang A, Detre JA. Neural correlates of voluntary and involuntary risk taking in the human brain: An fMRI study of the Balloon Analog Risk Task (BART). NeuroImage. 2008; 42(2):902-10. doi: 10.1016/j.neuroimage.2008.05.046 [DOI:10.1016/j.neuroimage.2008.05.046]
[17] Snaith RP, Hamilton M, Morley S, Humayan A, Hargreaves D, Trigwell P. A scale for the assessment of hedonic tone the snaithHamilton pleasure scale. British Journal of Psychiatry. 1995; 167(1):99-103. [DOI:10.1192/bjp.167.1.99]

[18] Millon T, Davis R. Millon clinical multiaxial inventory-II. Bloomington: Pearson Assessments; 1998. [PMID]

[19] Chegini M, Delavar A, Garaei B. [Psychometric properties Millon Clinical Multiaxial Inventoy (MCMI-III) (Persian)]. Quarterly Journal of Advanced Psychological Research. 2013; 8(29):135-162.

[20] Links PS, Heslegrave R, Reekum R van. Impulsivity: Core aspect of borderline personality disorder. Journal of Personality Disorders. 1999; 13(1):1-9. [DOI:10.1521/pedi.1999.13.1.1]

[21] Lawrence KA, Allen JS, Chanen AM. Impulsivity in borderline personality disorder: Reward-based decision-making and its relationship to emotional distress. Journal of Personality Disorders. 2010; 24(6):785-99. [DOI:10.1521/pedi.2010.24.6.785]

[22] Marissen MAE, Arnold N, Franken IHA. Anhedonia in borderline personality disorder and its relation to symptoms of impulsivity. Psychopathology. 2012; 45(3):179-84. [DOI:10.1159/000330893]

[23] Eysenck HJ. Biological basis of personality. Nature. 1963; 199(4898):1031-4. [DOI:10.1038/1991031a0]

[24] Gray JA. Brain systems that mediate both emotion and cognition. Cognition \& Emotion. 1990; 4(3):269-88. [DOI:10.1080/02699939008410799]

[25] Lempert KM, Pizzagalli DA. Delay discounting and futuredirected thinking in anhedonic individuals. Journal of Behavior Therapy and Experimental Psychiatry. 2010; 41(3):258-64. [DOI:10.1016/j.jbtep.2010.02.003]

[26] Schmahl CG, Elzinga BM, Ebner UW, Simms T, Sanislow C, Vermetten E, et al. Psychophysiological reactivity to traumatic and abandonment scripts in borderline personality and posttraumatic stress disorders: a preliminary report. Psychiatry Research. 2004; 126(1):33-42. [DOI:10.1016/j.psychres.2004.01.005]

[27] Herpertz SC, Kunert HJ, Schwenger UB, Sass H. Affective responsiveness in borderline personality disorder: A psychophysiological approach. American Journal of Psychiatry. 1999; 156(10):1550-6. [DOI:10.1176/ajp.156.10.1550] 